\title{
Entanglement of linear cluster states in terms of averaged entropies
}

\author{
CAO Ye ${ }^{1}$, LI Hui $^{1} \&$ LONG GuiLu ${ }^{1,2^{*}}$ \\ ${ }^{1}$ State Key Laboratory of Low-Dimensional Quantum Physics and Department of Physics, Tsinghua University, Beijing 100084, China; \\ ${ }^{2}$ Tsinghua National Laboratory for Information Science and Technology, Tsinghua University, Beijing 100084, China
}

Received August 15, 2012; accepted October 6, 2012

\begin{abstract}
An entanglement measure, multiple entropy measures (MEMS) was proposed recently by using the geometric mean of partial entropies over all possible $i$-body combinations of the quantum system. In this work, we study the average subsystem von Neumann entropies of the linear cluster state and investigated the quantum entanglement of linear cluster states in terms of MEMS. Explicit results with specific particle numbers are calculated, and some analytical results are given for systems with arbitrary particle numbers. Compared with other example quantum states such as the GHZ states and W states, the linear cluster states are "more entangled" in terms of MEMS, namely their averaged entropies are larger than the GHZ states and W states.
\end{abstract}

average subsystem entropies, cluster states, multiple entropy measures, quantum entanglement

Citation: Cao Y, Li H, Long G L. Entanglement of linear cluster states in terms of averaged entropies. Chin Sci Bull, 2013, 58: 48-52, doi: 10.1007/s11434-012-5604-2

Entanglement $[1,2]$ is one of the most salient properties of quantum systems. Quantum entanglement led to the experimental verification for Bell inequality which confirmed quantum mechanics [3]. In recent years, quantum entanglement has been one of the key driving forces that advanced the explosive development of quantum information and quantum computation [4-12]. Entanglement has developed from a philosophical concept into a frontier research paradigm, and much more attention has been paid to the quantification of entanglement. Two measures of entanglement, formation and distillation $[15,16]$, were proposed by Bennett et al. Partial von Neumann entropy [15,16], relative entropy [17, 18], concurrence [19-23] and other contributions [24-48] were also studied and recognized as "good" entanglement measures.

In studying quantum entanglement, the density matrices are often used. It should be mentioned that density matrix can represent different physical quantities. In the case of proper mixture, the density matrix represents the averaged "state" of an ensemble of "molecules", while the improper mixture describes the averaged "state" of a subsystem in a coupled quantum system. Though the mathematical expressions are the same, their physical properties are quite differ-

*Corresponding author (email: gllong@ mail.tsinghua.edu.cn) ent $[49,50]$. In studying quantum entanglement, we are actually using the improper mixture meaning of the density matrix. The reduced density matrix is obtained by tracing out other degrees of freedom of a composite quantum system. Based on separating the correlations encoded by a density matrix into a common set of marginals, Partovi [37] proposed a measurement in which $N ! / 2$ quantities are used to quantify an $N$-qubit system. In the case of $N \leqslant 3$, a "good" measurement [38] can be constructed from the arithmetic average entropy of single reduced density matrices. Realizing the lack of one-qubit reduction, Higuchi et al. [51,52] proposed using the arithmetic mean of two-particle entropies as a measure of entanglement, and reported on a four-qubit entangled state:

$$
\begin{aligned}
\left|M_{4}\right\rangle= & \sqrt{\frac{1}{6}}[|0011\rangle+|1100\rangle)+\omega(|1010\rangle+|0101\rangle \\
& \left.+\omega^{2}(|1001\rangle+|0110\rangle)\right], \omega=e^{2 i \pi / 3},
\end{aligned}
$$

which is more entangled than the four-qubit GHZ state.

More recently, Liu et al. [53] proposed multiple averaged entropy measures based on a vector with $m=[N / 2]$ components: $\left[S_{1}, S_{2}, \cdots, S_{m}\right]$, where the $S_{i}$ is the geometric mean of $i$-body partial entropy of the system 


$$
S_{n}=\left[\prod_{i_{1} i_{2} \cdots i_{n}}^{N}, E_{i_{1}, i_{2}, \cdots, i_{n}}\right]^{\frac{1}{C_{N}^{n}}} .
$$

$E_{i_{1}, i_{2}, \cdots, i_{n}}$ is the $i$-body von Neumann entropy for the $\left\{i_{1}, i_{2}, \cdots, i_{n}\right\}$ particles

$$
E_{i_{1} i_{2} \cdots i_{n}}=-\operatorname{Tr}\left[\left(\rho_{\Psi}\right)_{i_{1} \cdots i_{n}} \log _{2}\left(\rho_{\Psi}\right)_{i_{1} \cdots i_{n}}\right]
$$

where $\rho_{i_{1}, i_{2}, \cdots, i_{n}}$ is the reduced density matrix with the average of other $N-n$ particles and $C_{N}^{n}$ is the combination number

$$
C_{N}^{n}=\frac{N !}{(N-n) ! n !}
$$

The physical picture for $S_{i}$ can be understood in the following way. First, when $i$ equals 1 , it reflects the entanglement feature in terms of single particles. Later on, when $i \geqslant 2, S_{i}$ describes the entanglement of the $i$-body system as a whole with other $N-n$ particles. The upper bound for $E_{i_{1}, i_{2}, \cdots, i_{n}}$ is $n$, so if an $N$-particle state maximizes all the $E_{i_{1}, i_{2}, \cdots, i_{n}}, n=1,2, \cdots,[N / 2]$, it makes $S_{n}$ equals to $n$ and presents a maximally entangled state. For $N=2,3$, $[N / 2]=1$, the quantum states that satisfy $S_{1}=1$ are maximum entangled states. For $N=4$, Higuchi and Sudbery [51] proved that the ideal entangled state which maximizes all the $E_{i_{1}, i_{2}}$ does not exist. It should be noted that the MEMS constitutes only part of the entanglement measure. To describe quantum entanglement fully, more quantities are required in addition to the $N / 2$ MEMS quantities. While the GHZ states have smaller MEMS than the cluster state, they are bigger in other entanglement measures. For instance, GHZ states have the maximal violation of Bell inequality. This is the reason why a cluster state cannot be reduced to a GHZ state by local operation and classical communication. This is still an interesting and important issue being studied.

Liu et al. [53] studied the W state

$$
\left|W_{N}\right\rangle=\sqrt{\frac{1}{N}}\{|0 \cdots 01\rangle+\cdots+|10 \cdots 0\rangle\},
$$

and the analytic MEMS were obtained as

$$
S_{i}=\left[-\frac{N-i}{N} \log _{2} \frac{N-i}{N}-\frac{i}{N} \log _{2} \frac{i}{N}\right] .
$$

Obviously, all the $S_{i}$ are less than 1 . Hence, the W state is generally less entangled than the GHZ state in terms of multiple entropy measures (MEMS). The MEMS for the GHZstates were also obtained and all the GHZ-states $S_{i}$ are equal to 1 . Liu et al. [56] used MEMS and studied the entanglement properties of nine families four-qubit pure states which are classified by SLOCC [47].

In this work, we study the entangled properties of linear cluster state which was proposed by Briegel and Raussendorf for the purpose of persistent entanglement and used to construct a one-way quantum computer $[54,55]$.
The $N$-qubit linear cluster state [54] is defined as

$$
\left|C_{N}\right\rangle=\frac{1}{2^{N / 2}} \otimes\left(|0\rangle_{a} \sigma_{z}^{a+1}+|1\rangle_{a}\right)
$$

It holds a high persistency of entanglement which means that $\sim N / 2$ qubits have to be measured to eliminate the entanglement. Through the Schmidt decomposition, the state can be transformed into another clear form

$$
\left|C_{N}\right\rangle=\sum_{i_{1}, \cdots, i_{N}} \alpha_{i_{1}, \cdots, i_{N}}\left|i_{1}\right\rangle \cdots\left|i_{n}\right\rangle,
$$

where $i_{k}$ is 0 or $1, k=1, \cdots, N$. However, the expansion coefficients are not obvious. From the original definition of the linear cluster state, we can find that the expansion contains $2^{N}$ items. Under local unitary operation or basis transformation, some items cancel each other. After some tedious calculation, the simplified expressions for $\left|C_{8}\right\rangle,\left|C_{9}\right\rangle, \cdots,\left|C_{14}\right\rangle$ are obtained. The simplest expression of $\left|C_{N}\right\rangle$ holds $2^{[N / 2]}$ items.

First, we study the $S_{3}$ and $S_{4}$ of $\left|C_{8}\right\rangle$, whose simplest form is

$$
\begin{aligned}
\left|C_{8}\right\rangle= & |00101010\rangle+|00100111\rangle+|00010001\rangle \\
& +|00011100\rangle+|11001001\rangle+|11000100\rangle \\
& +|11110010\rangle+|11111111\rangle-|00101001\rangle \\
& -|00100100\rangle-|00010010\rangle-|00011111\rangle \\
& -|11001010\rangle-|11000111\rangle-|11110001\rangle \\
& -|11111100\rangle .
\end{aligned}
$$

There are $C_{8}^{3}=56$ combinations of the reduced positions for $S_{3}$. We traced all the possible conditions and listed the results in Table 1. It can be found that the entropies are all integers with separate values of 1,2 , and 3 . The result of 3 occurs 38 times, 2 occurs 16 times, and 1 occurs only 2 times. $S_{3}$ for the eight-qubit linear cluster state is $\sqrt[56]{2^{16} 3^{38}} \simeq 2.67$, which is slightly less than 3 .

Now it is interesting to check the $S_{4}$ of $\left|C_{8}\right\rangle$. After some numerical cyclic reductions, we found that the results of von Neumann entropy are also the same integers as $S_{3}$. There are four separate values, 1, 2, 3, and 4 . The result of 4 occurs 16 times, 3 occurs 36 times, 2 occurs 16 times, and 1 occurs 2 times. The occurrences of 1 and 2 are the same as $S_{3}$. The $S_{4}$ for $\left|C_{8}\right\rangle$ is $\sqrt[70]{2^{16} 3^{36} 4^{16}}=2^{\frac{\log _{2}\left(2^{16_{3} 3_{4} 16}\right)}{70}} \simeq 2.94$.

Next, we give the results of $S_{3}$ and $S_{4}$ for the nine-qubit linear cluster state

$$
\begin{aligned}
\left|C_{9}\right\rangle= & |001010101\rangle-|001010010\rangle-|001001001\rangle \\
& +|001001110\rangle-|000100101\rangle+|000100010\rangle \\
& -|111111001\rangle+|111111110\rangle+|000111001\rangle \\
& -|000111110\rangle-|110010101\rangle+|110010010\rangle \\
& +|110001001\rangle-|110001110\rangle+|111100101\rangle \\
& -|111100010\rangle .
\end{aligned}
$$

The simplest expression of $\left|C_{9}\right\rangle$ keeps $2^{[9 / 2]}$ direct product items, which verifies the formula $2^{[N / 2]}$ given obove. We list all the resources of $S_{3}$ for $\left|C_{9}\right\rangle$ in Table 2 . In the case of $S_{4}$, 
Table 1 Combinations and entropies of $\left|C_{8}\right\rangle$ for $S_{3}$. The numbers under the column "reduction" are those qubits that are being traced. The numbers in the column under "remain" are those qubits that are left over after tracing over the "reduction" qubits. The number under column "entropy" is the entropy of the reduced density matrix of the "remain" subsystem

\begin{tabular}{|c|c|c|c|c|c|}
\hline Reduction & Remain & Entropy & Reduction & Remain & Entropy \\
\hline 12345 & 678 & 1 & 12346 & 578 & 2 \\
\hline 12347 & 568 & 2 & 12348 & 567 & 2 \\
\hline 12358 & 467 & 3 & 12356 & 478 & 2 \\
\hline 12357 & 468 & 3 & 12378 & 456 & 2 \\
\hline 12368 & 457 & 3 & 12367 & 458 & 3 \\
\hline 12467 & 358 & 3 & 12457 & 368 & 3 \\
\hline 12478 & 356 & 3 & 12468 & 357 & 3 \\
\hline 12456 & 378 & 2 & 12458 & 367 & 3 \\
\hline 12578 & 346 & 3 & 12678 & 345 & 2 \\
\hline 12567 & 348 & 3 & 12568 & 347 & 3 \\
\hline 13568 & 247 & 3 & 13458 & 267 & 3 \\
\hline 13578 & 246 & 3 & 13678 & 245 & 3 \\
\hline 13468 & 257 & 3 & 13478 & 256 & 3 \\
\hline 13457 & 268 & 3 & 13567 & 248 & 3 \\
\hline 13456 & 278 & 2 & 13467 & 258 & 3 \\
\hline 14678 & 235 & 3 & 14578 & 236 & 3 \\
\hline 15678 & 234 & 2 & 14568 & 237 & 3 \\
\hline 14567 & 238 & 3 & 24567 & 138 & 3 \\
\hline 23467 & 158 & 3 & 24678 & 135 & 3 \\
\hline 24578 & 136 & 3 & 23457 & 168 & 3 \\
\hline 23478 & 156 & 3 & 23678 & 145 & 3 \\
\hline 25678 & 134 & 2 & 23567 & 148 & 3 \\
\hline 23578 & 146 & 3 & 23458 & 167 & 3 \\
\hline 23468 & 157 & 3 & 24568 & 137 & 3 \\
\hline 23456 & 178 & 2 & 23568 & 147 & 3 \\
\hline 35678 & 124 & 2 & 34578 & 126 & 2 \\
\hline 34678 & 125 & 2 & 45678 & 123 & 1 \\
\hline 34567 & 128 & 2 & 34568 & 127 & 2 \\
\hline
\end{tabular}

we just give the total number of each classification for the sake of space.

Using mathematical induction from the complex results obtained for the specific qubit numbers, we found that three types of subsystem von Neumann entropies are the same as in $\left|C_{8}\right\rangle$. The number of $E=3$ is $63, E=2$ is 19 , and $E=1$ is just 2. The $S_{3}$ for $\left|C_{9}\right\rangle$ is $\sqrt[84]{2^{19} 3^{63}}=2^{\frac{\log _{2}\left(2^{19} 3^{63}\right)}{84}} \simeq 2.74$. In the case of $S_{4}$, the number of $E=4$ is $48, E=3$ is $57, E=2$ is 19 and $E=1$ is $2 . S_{4}$ for $\left|C_{9}\right\rangle$ is $\sqrt[126]{2^{16} 3^{36} 4^{16}}=2^{\frac{\log _{2}\left(2^{19} 3^{57} 4^{48}\right)}{126}} \simeq$ 3.16 .

We studied the $S_{3}$ and $S_{4}$ for $N$ from 8 to 14, and arranged the results in Tables 3 and 4 , respectively. From the classification, we got the rules of $S_{3}$ and $S_{4}$, which are listed in eqs. (11) and (12), respectively. The number of $E=1$ is always 2 . By means of curve fitting, the numbers of $E=2$ and $E=3$ were found to increase linearly and quadratically.

The construction of $S_{3}$ component of MEMS for a $N$-qubit cluster state,

$$
\left\{\begin{array}{rl}
\text { Times } & \text { Entropy } \\
2 & E=1, \\
3 N-8 & E=2, \\
C_{N}^{3}-3 N+6 & E=3 .
\end{array}\right.
$$

Table 2 Combinations and entropies of $\left|C_{9}\right\rangle$ for $S_{3}$

\begin{tabular}{|c|c|c|c|c|c|}
\hline Reduction & Remain & Entropy & Reduction & Remain & Entropy \\
\hline 123456 & 789 & 1 & 123457 & 689 & 2 \\
\hline 123458 & 679 & 2 & 123459 & 678 & 2 \\
\hline 123469 & 578 & 3 & 123467 & 589 & 2 \\
\hline 123468 & 579 & 3 & 123489 & 567 & 2 \\
\hline 123479 & 568 & 3 & 123478 & 569 & 3 \\
\hline 123578 & 469 & 3 & 123568 & 479 & 3 \\
\hline 123589 & 467 & 3 & 123579 & 468 & 3 \\
\hline 123567 & 489 & 2 & 123569 & 478 & 3 \\
\hline 123689 & 457 & 3 & 123789 & 456 & 2 \\
\hline 123678 & 459 & 3 & 123679 & 458 & 3 \\
\hline 124679 & 358 & 3 & 124569 & 378 & 3 \\
\hline 124689 & 357 & 3 & 124789 & 356 & 3 \\
\hline 124579 & 368 & 3 & 124589 & 367 & 3 \\
\hline 124568 & 379 & 3 & 124678 & 359 & 3 \\
\hline 124567 & 389 & 2 & 124578 & 369 & 3 \\
\hline 125789 & 346 & 3 & 125689 & 347 & 3 \\
\hline 126789 & 345 & 2 & 125679 & 348 & 3 \\
\hline 125678 & 349 & 3 & 135678 & 249 & 3 \\
\hline 134578 & 269 & 3 & 135789 & 246 & 3 \\
\hline 135689 & 247 & 3 & 134568 & 279 & 3 \\
\hline 134589 & 267 & 3 & 134789 & 256 & 3 \\
\hline 136789 & 245 & 3 & 134678 & 259 & 3 \\
\hline 134689 & 257 & 3 & 134569 & 278 & 3 \\
\hline 134579 & 268 & 3 & 135679 & 248 & 3 \\
\hline 134567 & 289 & 2 & 134679 & 258 & 3 \\
\hline 146789 & 235 & 3 & 145689 & 237 & 3 \\
\hline 145789 & 236 & 3 & 156789 & 234 & 2 \\
\hline 145678 & 239 & 3 & 145679 & 238 & 3 \\
\hline 245679 & 138 & 3 & 234679 & 158 & 3 \\
\hline 246789 & 135 & 3 & 245689 & 137 & 3 \\
\hline 234569 & 178 & 3 & 234689 & 157 & 3 \\
\hline 234789 & 156 & 3 & 245789 & 136 & 3 \\
\hline 234579 & 168 & 3 & 234589 & 167 & 3 \\
\hline 235689 & 147 & 3 & 236789 & 145 & 3 \\
\hline 256789 & 134 & 2 & 235679 & 148 & 3 \\
\hline 235789 & 146 & 3 & 234578 & 169 & 3 \\
\hline 234568 & 179 & 3 & 234678 & 159 & 3 \\
\hline 245678 & 139 & 3 & 234567 & 189 & 2 \\
\hline 235678 & 149 & 3 & 356789 & 124 & 2 \\
\hline 345789 & 126 & 2 & 345689 & 127 & 2 \\
\hline 346789 & 125 & 2 & 456789 & 123 & 1 \\
\hline 345679 & 128 & 2 & 345678 & 129 & 2 \\
\hline
\end{tabular}

Table 3 Results of the three-body von Neumann entropy

\begin{tabular}{cccccccc}
\hline$N$ & 8 & 9 & 10 & 11 & 12 & 13 & 14 \\
\hline$E=1$ & 2 & 2 & 2 & 2 & 2 & 2 & 2 \\
$E=2$ & 16 & 19 & 22 & 25 & 28 & 31 & 34 \\
$E=3$ & 38 & 63 & 96 & 138 & 190 & 253 & 328 \\
\hline
\end{tabular}

Table 4 Results of the four-body von Neumann entropy

\begin{tabular}{cccccccc}
\hline$N$ & 8 & 9 & 10 & 11 & 12 & 13 & 14 \\
\hline$E=1$ & 2 & 2 & 2 & 2 & 2 & 2 & 2 \\
$E=2$ & 16 & 19 & 22 & 25 & 28 & 31 & 34 \\
$E=3$ & 36 & 57 & 82 & 111 & 144 & 181 & 222 \\
$E=4$ & 16 & 48 & 104 & 192 & 321 & 501 & 743 \\
\hline
\end{tabular}


The construction of $S_{4}$ component of MEMS for a $N$-qubit cluster state,

$$
\left\{\begin{array}{rl}
\text { Times } & \text { Entropy } \\
2 & E=1, \\
3 N-8 & E=2, \\
2 N^{2}-13 N+12 & E=3, \\
C_{N}^{4}-2 N^{2}+10 N-6 & E=4 .
\end{array}\right.
$$

By the definition of MEMS, we obtain the analytic results of $S_{3}$ and $S_{4}$ for a cluster state with $N$ qubits,

$$
\begin{aligned}
& S_{3}=\left[2^{3 N-8} 3^{C_{N}^{3}-3 N+8}\right]^{\frac{1}{C_{N}^{3}}}, \\
& S_{4}=\left[2^{3 N-8} 3^{2 N^{2}-13 N+12} 4^{C_{N}^{4}-2 N^{2}+10 N-6}\right]^{\frac{1}{C_{N}^{4}}} .
\end{aligned}
$$

We plot the $S_{3}$ and $S_{4}$ versus $N$ in Figures 1 and 2, respectively. The curves show that as $N$ increases, $S_{3}$ and $S_{4}$ tend toward the upper bound.

In summary, we used the MEMS entanglement measures to study the linear cluster state with focus on three-body and four-body average von Neumann entropies. All the data were obtained by numerical calculation for $N$ up to 14 . The results show that the linear cluster state is more entangled than the GHZ state and the W state in terms of the MEMS quantities. For large $N$ value, the $S_{3}$ and $S_{4}$ are close to 3 and 4, which are the upper bounds of the three- and four-body von Neumann entropy, respectively. Analytic results of $S_{3}$ and $S_{4}$

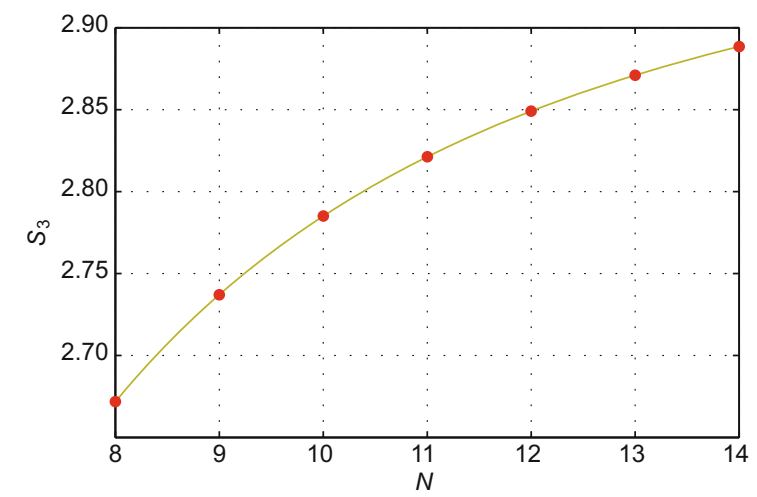

Figure 1 (Color online) $S_{3}$ versus $N$ for the linear cluster state.

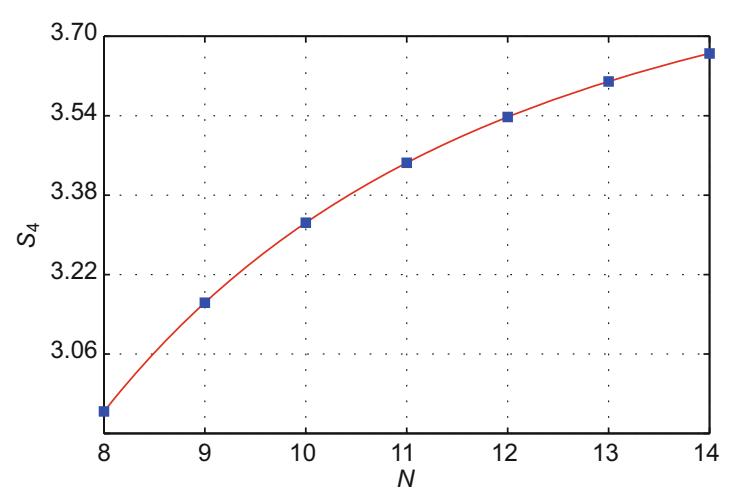

Figure 2 (Color online) $S_{4}$ versus $N$ for the linear cluster state. for arbitrary $N$ qubit linear cluster states are obtained, and explicit expressions for them are given. These results are helpful for understanding the entanglement nature of the linear cluster states.

We thank Prof. S. M. Fei for his helpful discussion. This work was supported by the National Natural Science Foundation of China (10874 098,11175094) and the National Basic Research Program of China (2009CB929402, 2011CB9216002).

1 Schoedinger E. Naturwissenschaften, 1935, 23: 807-849

2 Einstein A, Podolsky B, Rosen N. Phys Rev, 1935, 47: 777-780

3 Peres A. Phys Rev A, 1996, 54: 2685-2689

4 Nielsen M A, Chuang I L. Quantum Computation and Quantum Information. Cambridge: Cambridge University Press, 2000

5 Wang Y Z, Hou J C, Guo Y. Chin Sci Bull, 2012, 57: 1643-1647

6 Qian Y, Zhang Y Q, Xu J B. Chin Sci Bull, 2012, 57: 1637-1642

7 Salemian S, Mohammadnejad S. Chin Sci Bull, 2011, 56: 618-625

8 Li M, Fei S M, Li-Jost X Q. Chin Sci Bull, 2011, 56: 945-954

9 Ding S C, Jin Z. Chin Sci Bull, 2007, 52: 2161-2166

10 Ren X Z, Cong H L, Wang X W, et al. Sci China Phys Mech Astron, 2011, 54: 1625-1630

11 Li X K, Li J L, Liu B, et al. Sci China Phys Mech Astron, 2011, 54: 1471-1475

12 Chen C Y, Sun Q. Sci China Phys Mech Astron, 2011, 54: 930-935

13 Plenio M B, Virmani S. Quant Inf Comp, 2007, 7: 1-51

14 Horodecki R, Horodecki P, Horodecki M, et al. Rev Mod Phys, 2009, 81: 865-942

15 Bennett C H, Bernstein H J, Popescu S, et al. Phys Rev A, 1996, 53: 2046-2052

16 Bennett C H, DiVincenzo D P, Smolin J A, et al. Phys Rev A, 1996, 54: 3824-3851

17 Vedral V, Plenio M B, Rippin M R, et al. Phys Rev Lett, 1997, 78: 2275-2279

18 Vedral V, Plenio M B. Phys Rev A, 1998, 57: 1619-1633

19 Wootters W K. Phys Rev Lett, 1998, 80: 2245-2248

20 Uhlmann A. Phys Rev A, 2000, 62: 032307

21 Audenaert K, Verstraete F, De Moor B. Phys Rev A, 2001, 64: 052304

22 Fei S M, Jost J, Li-Jost X Q, et al. Phys Lett A, 2002, 310: 333-338

23 Fei S M, Li-Jost X Q. Rep Math Phys, 2004, 53: 195-210

24 Thapliyal A. Phys Rev A, 1999, 59: 3336-3342

25 Coffman V, Kundu J, Wootters W K. Phys Rev A, 2000, 61: 052306

26 Horodecki M, Horodecki M P, Horodecki R. Phys Rev Lett, 2000, 84: 2014-2017

27 Bennett C H, Popescu S, Rohrlich D, et al. Phys Rev A, 2000, 63: 012307

28 Acín A, Andrianov A, Costa L, et al. Phys Rev Lett, 2000, 85: 15601563

29 Dür W, Vidal G, Cirac J I. Phys Rev A, 2000, 62: 062314

30 Wu S J, Zhang Y D. Phys Rev A, 2000, 63: 012308

31 Li Y S, Zeng B, Liu X S, et al. Phys Rev A, 2001, 64: 054302

32 Schliemann J, Cirac J I, Kus M, et al. Phys Rev A, 2001, 64: 022303

33 Paskauskas R, You L. Phys Rev A, 2001, 64: 042310

34 Sudbery A. J Phys A, 2001, 34: 643-652

35 Rajagopal A K, Rendell R W. Phys Rev A, 2002, 65: 032328

36 Rajagopal A K, Rendell R W. Phys Rev A, 2002, 66: 022104

37 Partovi M H. Phys Rev Lett, 2004, 92: 077904

38 Pan F, Liu D, Lu G, et al. Int J Theor Phys, 2004, 43: 1241-1247

39 Pan F, Liu D, Lu G Y, et al. Phys Lett A, 2005, 336: 384-389 
40 Cao W C, Liu D, Pan F, et al. Sci China Ser G: Phys Mech Astron, 2006, 49: 606-615

41 Yu S X, Pan J W, Chen Z B, et al. Phys Rev Lett, 2003, 91: 217903

42 Zhou D L, Zeng B, Xu Z, et al. Phys Rev A, 2006, 74: 052110

43 Chen Z Q. Phys Rev Lett, 2004, 93: 110403

44 Chen J L, Wu C F, Kwek L C, et al. Phys Rev Lett, 2004, 93: 140407

45 Cai J M, Zhou Z W, Zhou X X, et al. Phys Rev A, 2006, 74: 042338

46 Facchi P, Florio G, Pascazio S. Phys Rev A, 2006, 74: 042331

47 Verstraete F, Dehaene J, De Moor B, et al. Phys Rev A, 2002, 65: 052112
48 Osterloh O, Siewert J. Phys Rev A, 2005, 72: 012337

49 d'Espagnat B. Veiled Reality: An Analysis of Present-day Quantum Mechanical Concepts. Reading, Massachuetts: Addison-Wesley, 1995

50 Long G L, Zhou Y F, Jin J Q, et al. Found Phys, 2006, 36: 1217-1243

51 Higuchi A, Sudbery A. Phys Lett A, 2000, 273: 213-217

52 Brierley S, Higuchi A. J Phys A: Math Theor, 2007, 40: 8455-8465

53 Liu D, Zhao X, Long G L. Commun Theor Phys, 2010, 54: 825-828

54 Briegel H J, Raussendorf R. Phys Rev Lett, 2001, 86: 910-913

55 Raussendorf R, Briegel H J. Phys Rev Lett, 2001, 86: 5188-5191

56 Liu D, Zhao X, Long G L. Commun Theor Phys, 2008, 49: 329-332

Open Access This article is distributed under the terms of the Creative Commons Attribution License which permits any use, distribution, and reproduction in any medium, provided the original author(s) and source are credited. 